\title{
Correlation between B-Type Natriuretic Peptide and Functional/Cognitive Parameters in Discharged Congestive Heart Failure Patients
}

\author{
Laura Leto, ${ }^{1}$ Marzia Testa, ${ }^{1}$ and Mauro Feola ${ }^{2}$ \\ ${ }^{1}$ School of Geriatrics, University of Medicine, 10100 Turin, Italy \\ ${ }^{2}$ Cardiovascular Rehabilitation-Heart Failure Unit, Ospedale SS Trinita, 12045 Fossano, Italy \\ Correspondence should be addressed to Mauro Feola; m_feola@virgilio.it
}

Received 10 December 2014; Accepted 26 March 2015

Academic Editor: Kristin Eckardt

Copyright (C) 2015 Laura Leto et al. This is an open access article distributed under the Creative Commons Attribution License, which permits unrestricted use, distribution, and reproduction in any medium, provided the original work is properly cited.

The determination of B-type natriuretic peptides (BNP) may have a role in the diagnosis of heart failure (HF) or guiding HF therapy. This study investigated the role of BNP determination in a cohort of elderly patients admitted to hospital with acute decompensated HF and its correlation with main demographic, clinical, and instrumental data and evaluated possible association with major outcome such as mortality or readmission after a 6-month period of follow-up. Methods. From October 2011 to May 2014 consecutive patients admitted to our unit with symptoms of acute HF or worsening of chronic HF entered the study collecting functional, echocardiographic, and hydration parameters. Correlation between BNP and main parameters was analysed, as well as the mortality/6-month readmission rate. Results. In 951 patients (mean age $71 \mathrm{ys;} 37 \%$ females) a positive correlation was obtained between BNP and age, creatinine levels, NYHA class at admission and discharge, and levels of hydration; an inverse, negative correlation between BNP and sodium levels, LVEF, distance performed at 6MWT at admission and at discharge, and scores at MMSE at admission and discharge emerged. BNP levels at admission and at discharge were furthermore clearly associated with mortality at 6 months (Chi-square 704.38, $p=0.03$ ) and hospital readmission (Chi-square 741.57, $p<0.01$ ). Conclusion. In an elderly HF population, BNP is related not only with clinical, laboratory, and instrumental data but also with multidimensional scales evaluating global status; higher BNP levels are linked with a worse prognosis in terms of mortality and 6-month readmission.

\section{Introduction}

B-type natriuretic peptide (BNP) is a 32-amino acid peptide primarily secreted by the cardiac ventricles in response to increased myocardial wall stress due to volume overload or higher end-diastolic pressure inside the ventricle itself. BNP has a diuretic, natriuretic, and vasodilating effect. BNP levels rise primarily in the presence of left ventricular dysfunction: the most recent guidelines suggest measuring it to support clinical decision making regarding the diagnosis of heart failure (HF), especially when diagnosis is uncertain [1]. The determination of natriuretic peptides (NP) may have a role also to guide HF therapy [1]. Both HF with reduced ejection fraction (EF) and HF with preserved $\mathrm{EF}$ can be associated with increased BNP levels, but also other clinical cardiac and noncardiac conditions can be linked with higher NP concentrations. Increasing levels of NP predict worse prognosis in a linear fashion across all stages of HF [2]; furthermore, decrease of NP concentrations is associated with a better prognosis than failure to decrease or increase [3]. However, no definitive data are established about the utility of a serial determination of NP levels in decreasing mortality or readmission. The present study aimed to investigate the role of BNP determination in a cohort of elderly patients admitted to hospital with acute decompensated HF and its correlation with main demographic, clinical, and instrumental data and to evaluate possible association with major outcome such as mortality or readmission after a 6-month period of follow-up.

\section{Patients and Methods}

From October 2011 to May 2014 consecutive patients admitted to our unit with symptoms of acute HF or worsening of 
chronic HF were asked to enter the study. HF was defined according to the presence of two major criteria or one major criterion and two minor criteria of the Framingham classification. Etiology of HF was researched and its severity according to NYHA (New York Heart Association) functional class was recorded; an echocardiogram was performed in all patients. A complete blood test was obtained; creatinine clearance according to Cockroft-Gault formula was calculated. Levels of BNP were measured at admission in all patients; 403 subjects underwent a BNP evaluation also at discharge. Body mass index (BMI) was calculated as weight in kilograms divided by the square of height in meters.

Hydration status was evaluated through a bioimpedance technique while performance was analysed with 6-minute walking test at discharge after compensation was achieved.

2.1. Doppler Echocardiography. Echocardiograms were performed with a Vivid 7 computed sonography system (GE Medical Systems, Waukesha, Wisconsin, USA) according to the recommendations of the American Society of Echocardiography. Two-dimensional apical 2- and 4-chamber views were used for volume measurements; left ventricular ejection fraction (LVEF) was calculated with modified Simpson's method using biplane apical (2- and 4-chamber) views. The LV end-diastolic volume and the LV end-systolic volume were recorded. All the echo examinations were performed by expert operators blinded to the results of BNP assay; the intraobserver variability in the evaluation of LVEF was found to be $<5 \%$. Echocardiographic measurements including LV end-diastolic diameter and the diastolic thickness of the ventricular septum and the posterior LV wall were determined according to the American Society Echocardiography recommendations [4]. Systolic dysfunction was defined as a level of LVEF $<50 \%$. The definition of restrictive filling pattern (grade 3) was a predefined modification of classifications used in prior studies: $E / A \geq 2$, DT $\leq 150 \mathrm{msec}, S / D$ ratio $<1$, and $A R>35 \mathrm{~cm} / \mathrm{sec}$. All these criteria should be verified to define the restrictive filling pattern. The other diastolic filling patterns were classified as follows: grade 1 (abnormal relaxation) when $E / A<1$ with DT $>240 \mathrm{~ms}$; grade 2 (pseudonormal) when $E / A$ is between 0.75 and 1.5 , DT is between 160 and $240 \mathrm{~ms}$, and finally $E / E a>15$ [5]. The presence of this diastolic pattern with LVEF $\geq 50 \%$ was defined as an isolated diastolic dysfunction.

2.2. BNP Assay. All blood samples were collected by venopuncture and immediately analysed with the bedside Triage Btype natriuretic fluorescence immunoassay (Biosite Diagnostics, La Jolla, CA, USA). The Triage Meter is used to measure BNP concentration by detecting a fluorescent emission that reproduces the amount of BNP in the blood. Two hundred and fifty $\mu \mathrm{L}$ of whole blood was added to the disposable device; then the cells were filtered and separated from the plasma with BNP, which entered a reaction chamber, containing fluorescent BNP antibodies. After 2-min incubation, the BNP-antibody mixture migrated to an area containing immobilised antibodies and remained fixed. The unbound fluorescent antibodies were washed away by the excess sample fluid. Then, the Triage Meter measured the fluorescent intensity of the BNP assay area. The assay results were complete in 15 minutes.

2.3. Six-Minute Walk Test. The 6MWT was performed in the discharging day according to the ATS Statement of the American Thoracic Society [6]. CHF patients able to walk underwent $6 \mathrm{MWT}$ if they did not meet the exclusion criteria (unstable angina and myocardial infarction during the previous month, resting heart rate $>120$, systolic blood pressure $>180 \mathrm{mmHg}$, or diastolic blood pressure $>100 \mathrm{mmHg}$ ).

2.4. Bioelectrical Impedance Vectorial Analysis (BIVA). Assessment of body fluid status was carried out using an electrical impedance analyser and BodyGram 2.1 software (Akern Pontassieve, Florence, Italy). The bioelectrical parameters of resistance, reactance, and phase angle were obtained with an electric alternating current flux of 800 microA and an operating frequency of $50 \mathrm{kHz}$. Whole body impedance measurements were taken by using a standard position of outer and inner electrodes on the right hand and foot. The entire procedure was performed according to the indications of the National Institutes of Health Technology assessment conference statements [7]. Bioelectrical impedance evaluates some basic properties of the body by measuring resistance, reactance (a form of opposition that electronic components exhibit to the passage of alternating current counterpart of direct current and indicates an absolute amount of body cell mass). Bioelectrical impedance is normally used to estimate the volumes of body fluid compartments allowing determining total body water and the ratio between extracellular and total body water. Resistance and reactance were always corrected for the patients' height. The clinical support of this method has been implemented using the vector analysis (Bioelectrical Impedance Vector Analysis, BIAVECTOR) [8]. The backward or forward position of parallel vectors to the major axis of ellipses is normally correlated with dehydration or hyperhydration. The normal value of hydration has been set at value $73.3 \%$ and the area within +1 SD was considered a satisfactory criterion for discharging CHF patients.

Functional status was evaluated through validated tools as activities of daily living (ADL) index [9], developed by Katz, which explores the level of dependence or independence in six basic tasks, that is, bathing, toileting, dressing, continence, movement, and feeding, Barthel index [10] exploring ADL functions and mobility, and instrumental activities of daily living (IADL) index [11] that involves more complex abilities such as use of phone, doing shopping, use of transports, and drugs management.

All patients underwent a complete examination of cognitive status with Mini Mental State Examination (MMSE) [12] while the presence of mood disorders was explored with Geriatric Depression Scale (GDS) [13]. The MMSE is an easy test that takes approximately 10 minutes to be administered and that covers seven different cognitive domains: temporal and spatial orientation, immediate and delayed memory, attention, calculation, and language (denomination, repetition, comprehension, reading, writing, and constructional apraxia). The maximum score is 30 and scores of $25-30$ are 
considered normal; total scores from 21 to 24 indicate mild impairment, from 10 to 20 moderate impairment, and $<10$ severe impairment. A correction can be applied according to age and years of schooling. The GDS is a 15 -item selfreport measure of depression in older adults; the participants are asked to answer "yes" or "no" according to how they felt in the past week. No questions on semantic expression of depressed mood are involved. Ten questions indicate the presence of depression when answered positively, while the rest indicate depression when answered negatively; one point is assigned to each answer if the presence of depression is suggested. Scores of $0-4$ are considered normal, 5-8 indicate mild depression, 9-11 indicate moderate depression, and 12-15 indicate severe depression. It takes approximately 57 minutes to be administered and its simplicity and rapid application enable the scale to be used with ill or moderately cognitively impaired individuals. The Minnesota Living with Heart Failure Questionnaire (MLWHFQ) [14] was used to assess quality of life in this population: it measures the effects of symptoms, functional limitations, and psychological distress on an individual quality of life. It consists of questions that assess the impact of frequent physical symptoms, the effects of HF on physical/social functions, and side effects of treatments, hospital stays, and costs of care.

2.5. Clinical Follow-Up. Death by any cause, cardiac transplantation, and worsening heart failure requiring readmission to the hospital were considered cardiovascular events. Data regarding the occurrence of cardiovascular events were collected from multiple sources in all patients.

2.6. Statistical Analysis. Counts and percentages for categorical and ordinal variables were calculated while continuous variables were expressed as mean \pm standard deviation (SD). BNP levels were evaluated both as a continuous variable and as a categorical variable (based on cut-off values). According to the plasma BNP value 2 groups were created: $<300 \mathrm{pg} / \mathrm{mL}$ and $\geq 300 \mathrm{pg} / \mathrm{mL}$ in order to evaluate the role of different levels of plasma BNP on clinical, laboratory, and instrumental parameters and the occurrence of cardiac events in the follow-up. Pearson's correlation coefficient was calculated to describe the linear correlation between BNP levels and multiple parameters. The comparison between different means in the two BNP groups was verified through the independent two-sample $t$-test (Student's $t$-test). Pearson's Chi-square test was used to evaluate the association between BNP levels and categorical outcome variables (mortality, hospital readmission). A normal distribution was assumed. A $p$ value $<0.05$ was considered significant. Analyses were performed using SPSS software per Windows, release 22.0, SPSS Inc., Chicago, USA.

\section{Results}

Data on 951 patients were recorded in the stated period. Table 1 shows main demographic, clinical, and instrumental data of the sample. Mean age was 70.8 years, and $74.2 \%$ of the sample was older than 65 years while $36.8 \%$ was older than 75. More than one-third of the sample was female. In $46.8 \%$ the etiology of heart failure was ischaemic, 96 subjects underwent previous percutaneous angioplasty, and 251 underwent by-pass graft. An automatic implantable cardioverter defibrillator (AICD) was implanted in $8.6 \%$ of the sample. In more than half of the examined population a LVEF lower than $50 \%$ emerged. Functional status was minimally impaired with a mean score at ADL index of 5.4 lost functions and a total Barthel score of 86.3. Patients in the sample presented a minimally compromised cognitive function (total score at MMSE of 27.3) and a depressed mood (total score at GDS of 6.4 on a 15 -item scale). In the 6 -month period of follow-up, 76 patients died while 57 (6\% of the sample) underwent a new hospital readmission. Mean BNP level at admission was $549.1 \mathrm{pg} / \mathrm{mL}$, and at discharge it was $408.9 \mathrm{pg} / \mathrm{mL}$; in 403 patients BNP levels were measured both at admission and at discharge: in $326(80.9 \%)$ they were lower at discharge while in 77 subjects no difference or even higher levels were present at discharge.

At univariate analysis, a positive correlation was obtained between BNP and age $(r=0.15 ; p<0.01)$, creatinine levels $(r=0.29 ; p<0.01)$, NYHA class at admission and discharge $(r=0.36 ; p<0.01 ; r=0.21 ; p<0.01)$, and levels of hydration at bioimpedance analysis $(r=0.37 ; p<0.01)$; an inverse, negative correlation between BNP and sodium levels $(r=-0.15 ; p<0.01)$, LVEF $(r=-0.46 ; p<0.01)$, scores at ADL index $(r=-0.20 ; p<0.01)$ and Barthel index $(r=-0.18 ; p<0.01)$, distance performed at 6MWT at admission and at discharge $(r=-0.25 ; p<0.01$ and $r=-0.30 ; p<0.01)$, and scores at MMSE at admission and discharge $(r=-0.17 ; p=0.02)$ emerged. Patients with BNP level higher than $300 \mathrm{pg} / \mathrm{mL}$ were older, with a worse renal function, a lower haemoglobin count, lower LVEF, a worse functional status measured with Barthel index, a higher hydration level assessed with bioimpedance analysis, a worse physical (at 6MWT) and cognitive (at MMSE) performance, and a depressed mood (at GDS), and experienced longer hospital stay (Table 2).

BNP levels were significantly lower in patients with HF with preserved LVEF compared to patients with HF with reduced LVEF (303.1 pg/mL versus $839.1 \mathrm{pg} / \mathrm{mL}, p<0.01$ ). BMI was calculated in 90 subjects: mean value was 25.5 and 36 patients (40\%) were overweight while $13.3 \%$ of the sample was obese. Overweight $(\mathrm{BMI}>25)$ and obese $(\mathrm{BMI}>$ 30) patients have lower BNP levels even if no statistical significance was reached, also because of the small sample size. In patients with diabetes, lower BNP concentrations were recorded $(1049 \mathrm{pg} / \mathrm{mL}$ versus $1414 \mathrm{pg} / \mathrm{mL})$ even if no statistical significance emerged (Table 3 ).

BNP levels at admission and at discharge were furthermore clearly associated with mortality at 6 months (Chisquare 704.38, $p=0.03$ ) and hospital readmission (Chisquare $741.57, p<0.01$ ).

\section{Discussion}

$\mathrm{BNP}$ is secreted by the cardiac ventricles in response to increased myocardial wall stress due to volume overload or higher end-diastolic pressure inside the ventricle itself. 
TABLE 1: Main demographic, clinical, and laboratory data.

\begin{tabular}{|c|c|c|}
\hline Clinical features & Patients $(n=951)$ & Range \\
\hline Age (years) & $70.8 \pm 10.3$ & $30-92$ \\
\hline Female & $352(37 \%)$ & \\
\hline $\begin{array}{l}\text { Mean ejection fraction }(\%) \\
\text { (i) Ejection fraction } \leq 50 \%\end{array}$ & $\begin{array}{c}46.9 \pm 14.4 \\
548(57.6 \%)\end{array}$ & $10-89$ \\
\hline \multicolumn{3}{|l|}{ Heart failure severity (NYHA class at admission) } \\
\hline (i) NYHA II & $388(40.8 \%)$ & \\
\hline (ii) NYHA III & $326(34.3 \%)$ & \\
\hline (iii) NYHA IV & $90(9.5 \%)$ & \\
\hline BNP level at admission (pg/mL) & $549.1 \pm 898.2$ & $4-17860$ \\
\hline Creatinine plasma level (mg/dL) & $1.17 \pm 0.6$ & $0.4-6.3$ \\
\hline $\begin{array}{l}\text { Creatinine clearance (according to Cockroft-Gault equation) } \\
(\mathrm{mL} / \mathrm{min})\end{array}$ & $62.3 \pm 25.4$ & $15-129$ \\
\hline Sodium serum level (mg/dL) & $139.6 \pm 7.3$ & $120-152$ \\
\hline Body mass index $(\mathrm{BMI})(n=90)$ & $25.5 \pm 5.4$ & $15-39$ \\
\hline \multicolumn{3}{|l|}{ Heart failure etiology } \\
\hline (i) Coronary artery disease & $445(46.8 \%)$ & \\
\hline (a) Previous by-pass graft surgery & $251(26.4 \%)$ & \\
\hline (b) Previous percutaneous revascularization & $96(10.1 \%)$ & \\
\hline (ii) Valvular cardiomyopathy & $258(43.7 \%)$ & \\
\hline Atrial fibrillation & $199(21 \%)$ & \\
\hline AICD & $82(8.6 \%)$ & \\
\hline 6-minute walking test (meters) & $310.7 \pm 93.0$ & $50-570$ \\
\hline Barthel index & $86.3 \pm 23.4$ & $0-100$ \\
\hline Score on MMSE & $27.3 \pm 3.2$ & $10-30$ \\
\hline GDS 15 items & $6.4 \pm 3.5$ & $0-15$ \\
\hline
\end{tabular}

NYHA: New York Heart Association; BNP: B-type natriuretic peptide; AICD: automatic implantable cardioverter defibrillator; MMSE: Mini Mental State Examination; GDS: Geriatric Depression Scale.

TABLE 2: Differences in clinical, laboratory, and instrumental parameters according to BNP levels.

\begin{tabular}{|c|c|c|c|}
\hline & $\mathrm{BNP} \geq 300 \mathrm{pg} / \mathrm{mL}$ & $\mathrm{BNP}<300 \mathrm{pg} / \mathrm{mL}$ & $p$ \\
\hline Age (years) & $72.4 \pm 9.6$ & $69.3 \pm 10.7$ & $<0.001$ \\
\hline Creatinine level (mg/dL) & $1.3 \pm 0.7$ & $1.0 \pm 0.4$ & $<0.001$ \\
\hline Creatinine clearance $(\mathrm{mL} / \mathrm{min})$ & $55.7 \pm 23.7$ & $70.2 \pm 25.3$ & $<0.001$ \\
\hline Haemoglobin $(g / d L)$ & $11.4 \pm 1.8$ & $12.2 \pm 2.0$ & 0.001 \\
\hline $\operatorname{LVEF}(\%)$ & $40.7 \pm 14.7$ & $53.1 \pm 11.2$ & $<0.001$ \\
\hline Barthel index at admission (score) & $81.2 \pm 26.0$ & $91.1 \pm 19.7$ & $<0.001$ \\
\hline Hydration level at BIVA at admission (\%) & $77 \pm 5.3$ & $73.9 \pm 2.4$ & $<0.001$ \\
\hline $6 \mathrm{MWT}$ at admission (meters) & $297.6 \pm 90.1$ & $321.8 \pm 94.2$ & 0.015 \\
\hline MMSE (score) & $26.7 \pm 3.6$ & $28.0 \pm 2.5$ & $<0.001$ \\
\hline GDS (score) & $8.2 \pm 4.7$ & $6.9 \pm 3.3$ & 0.046 \\
\hline Length of hospital stay (days) & $13.3 \pm 8.1$ & $11.1 \pm 7.2$ & $<0.001$ \\
\hline
\end{tabular}

LVEF: left ventricular ejection fraction; BIVA: bioelectrical impedance vectorial analysis; 6MWT: 6-minute walking test; MMSE: Mini Mental State Examination; GDS: Geriatric Depression Scale.

The activation of BNP gene in cardiomyocytes produces a precursor propeptide $\left(\right.$ proBNP ${ }_{108}$ ) further cleaved into the biologically inert amino terminal fragment (NT-proBNP) and the biologically active BNP. BNP has a diuretic and natriuretic effect increasing the glomerular filtration rate and decreasing sodium reabsorption in the collecting duct thus improving sodium excretion (downregulation of the renninangiotensin-aldosterone system); furthermore it decreases peripheral vascular resistance and increases smooth cells relaxation thus resulting in a vasodilating effect. In our 
TABLE 3: Differences in BNP levels in overweight or obese and normal weight patients and in patients with or without diagnosis of diabetes.

\begin{tabular}{lcc}
\hline & $\begin{array}{c}\text { BNP levels } \\
(\mathrm{pg} / \mathrm{mL})\end{array}$ & $p$ \\
\hline Overweight and obese $(\mathrm{BMI} \geq 25)$ & $570.4 \pm 678.3$ & 0.13 \\
Normal weight $(\mathrm{BMI}<25)$ & $1288.1 \pm 878.7$ & \\
\hline Presence of diabetes & $1049.2 \pm 1240.1$ & 0.53 \\
\hline Absence of diabetes & $1414.1 \pm 1875.1$ & \\
\hline
\end{tabular}

BNP: B-type natriuretic peptide; BMI: body mass index.

sample, sodium levels were lower in subjects with higher BNP concentration even if no statistical significance was reached (data not shown).

NT-proBNP and BNP differ also for half-life (120 versus 20 minutes) thus resulting in higher levels of circulating NTproBNP; they are both cleared by the kidney [15]. BNP levels rise primarily in the presence of left ventricular dysfunction: the most recent clinical guidelines on heart failure (HF) management suggest its measurement to support clinical decision regarding the diagnosis and to establish prognosis or disease severity in ambulatory patients with dyspnea [16], in chronic HF [17], in hospitalized subjects $[18,19]$, and in acute decompensated HF (ADHF) [20] (Class I, Level of Evidence A). According to ACCF/AHA guidelines [1], natriuretic peptide- (NP-) guided HF therapy can be useful to achieve optimal dosing of guideline-directed medical therapy in euvolemic patients followed in a structured disease management program [21-24] (Class IIa, Level of Evidence B) but it is not well established for ADHF [25] (Class IIb, Level of Evidence C). Furthermore, the usefulness of serial measurement of BNP or NT-proBNP to reduce hospitalization or mortality in patients with HF is not well established $[11,12,26]$ (Class IIb, Level of Evidence B). Both systolic dysfunction and HF with preserved ejection fraction (HFpEF) may be linked to elevated BNP and NT-proBNP levels, although HFpEF may be associated with lower concentrations of both peptides. Also in our sample of elderly hospitalized patients with ADHF, BNP levels were significantly lower in patients with HFpEF compared to patients with HF with reduced LVEF. However, also other conditions are associated with higher BNP levels: cardiac disease such as acute coronary syndrome, valvular heart disease [27], pericardial disease, atrial fibrillation [28], myocarditis, cardiac surgery and noncardiac ones such as renal failure [29], liver cirrhosis with ascites, pulmonary hypertension, and severe pneumonia. Advancing age may be linked with higher concentrations also without overt HF [30]; on the contrary obesity may result in lower BNP or NTproBNP serum levels even when $\mathrm{HF}$ is present $[31,32]$. In our sample BNP levels were positively associated with age; furthermore, overweight and obese patients had lower BNP concentrations if compared to subjects with BMI $<25$.

In our sample of ADHF patients, BNP was related to functional, cognitive, and behavioral scales that are part of a multidimensional approach usually applied in elderly comorbid patients in order to assess global status. BNP has consistent data as regards the relationship with cognitive impairment in general elderly population $[33,34]$, in individuals with cardiovascular disease [35], and in patients with $\mathrm{HF}$ $[36,37]$. In a cohort of 464 individuals, mean age of 79 years, MMSE was administered at baseline and after a follow-up period of 5 years: BNP was the only variable connected with decline of MMSE over time and it was associated with new diagnosis of dementia, defined according to DSM-IV criteria and to guidelines with an OR $=1.53$ (95\% CI 1.09-2.16, $p=$ 0.013) [33]. In the Rancho Bernardo Study, a populationbased study of the epidemiology of chronic disease on 950 older individuals with an average age of 77 years, elevated NT-proBNP values were independently associated with poor cognitive function on MMSE (OR 2.0, 95\% CI 1.1-3.6, and $p=$ 0.02) [34]. Regarding individuals with HF, in sixty patients hospitalized for exacerbation of HF (mean age 65.5 years, mean LVEF 32.9, and average BNP plasma level $683.3 \mathrm{pg} / \mathrm{mL}$ ) $\mathrm{BNP}$ was related with MMSE $(r=0.12, p=0.02)$ but not with other cognitive tests [35-37]. In our sample of ADHF patients with an almost normal mean MMSE score, BNP concentration was related with total MMSE score with subjects with higher NP levels experiencing more cognitive decline.

As for prognosis, increasing levels of NP were showed to predict worse prognosis in a linear fashion across all stages of HF [2]. Determination of NP plasmatic levels may be important both as an initial evaluation and during followup: according to observational studies a $30 \%$ decrease of NP levels is associated with a better prognosis than failure to decrease or increase [3]. In our sample BNP levels at admission and at discharge were related to a worse prognosis as for mortality and readmission at 6-month follow-up.

\section{Conclusion}

In conclusion, in an elderly HF population, hospitalized with signs and symptoms of congestive HF (more than a third of the sample females, with a moderate neurohormonal activation and more than half of the sample presenting an impaired LVEF), BNP is related not only with clinical, laboratory, and instrumental data that are usually assessed in HF patients but also with multidimensional scales evaluating global status; furthermore, also in this large single-centre population, higher BNP levels are linked with a worse prognosis in terms of mortality and readmission at 6 months.

\section{Conflict of Interests}

The authors declare that there is no conflict of interests regarding the publication of this paper.

\section{References}

[1] C. W. Yancy, M. Jessup, B. Bozkurt et al., "2013 ACCF/AHA guideline for the management of heart failure: a report of the American college of cardiology foundation/american heart association task force on practice guidelines," Journal of the American College of Cardiology, vol. 62, no. 16, pp. e147-e239, 2013.

[2] A. Sachdeva, T. B. Horwich, and G. C. Fonarow, "Comparison of usefulness of each of five predictors of mortality and urgent transplantation in patients with advanced heart failure," 
American Journal of Cardiology, vol. 106, no. 6, pp. 830-835, 2010.

[3] P. Bettencourt, A. Azevedo, J. Pimenta, F. Friões, S. Ferreira, and A. Ferreira, "N-terminal-pro-brain natriuretic peptide predicts outcome after hospital discharge in heart failure patients," Circulation, vol. 110, no. 15, pp. 2168-2174, 2004.

[4] D. J. Sahn, A. DeMaria, J. Kisslo, and A. Weyman, "Recommendations regarding quantitation in M-mode echocardiography: results of a survey of echocardiographic measurements," Circulation, vol. 58, no. 6, pp. 1072-1083, 1978.

[5] M. J. Garcia, J. D. Thomas, and A. L. Klein, "New doppler echocardiographic applications for the study of diastolic function," Journal of the American College of Cardiology, vol. 32, no. 4, pp. 865-875, 1998.

[6] ATS Committee on Proficiency Standards for Clinical Pulmonary Function Laboratories, "ATS statement: guidelines for the six-minute walk test," The American Journal of Respiratory and Critical Care Medicine, vol. 166, no. 1, pp. 111-117, 2002.

[7] "Bioelectrical impedance analysis in body composition measurement. Proceedings of a National Institutes of Health Technology Assessment Conference. Bethesda, Md, USA, December 1994," The American Journal of Clinical Nutrition, vol. 64, supplement, pp. 387S-532S, 1996.

[8] A. Piccoli, "Patterns of bioelectrical impedance vector analysis: learning from electrocardiography and forgetting electric circuit models," Nutrition, vol. 18, no. 6, pp. 520-521, 2002.

[9] T. F. Katz, "A.D.L. Activities of daily living," The Journal of the American Medical Association, vol. 185, pp. 914-919, 1963.

[10] F. I. Mahoney and D. W. Barthel, "Functional evaluation: the Barthel index," Maryland State Medical Journal, vol. 14, pp. 6165, 1965.

[11] M. P. Lawton and E. M. Brody, "Assessment of older people: self-maintaining and instrumental activities of daily living," Gerontologist, vol. 9, no. 3, pp. 179-186, 1969.

[12] M. F. Folstein, S. E. Folstein, and P. R. McHugh, "Mini-mental state. A practical method for grading the cognitive state of patients for the clinician," Journal of Psychiatric Research, vol. 12, no. 3, pp. 189-198, 1975.

[13] J. A. Yesavage, T. L. Brink, T. L. Rose et al., "Development and validation of a geriatric depression screening scale: a preliminary report," Journal of Psychiatric Research, vol. 17, no. 1, pp. 37-49, 1982.

[14] T. S. Rector and J. N. Cohn, "Assessment of patient outcome with the Minnesota Living with Heart Failure questionnaire: reliability and validity during a randomized, double-blind, placebo-controlled trial of pimobendan," The American Heart Journal, vol. 124, no. 4, pp. 1017-1025, 1992.

[15] S. M. Green, A. Martinez-Rumayor, S. A. Gregory et al., "Clinical uncertainty, diagnostic accuracy, and outcomes in emergency department patients presenting with dyspnea," Archives of Internal Medicine, vol. 168, no. 7, pp. 741-748, 2008.

[16] R. S. Vasan, E. J. Benjamin, M. G. Larson et al., "Plasma natriuretic peptides for community screening for left ventricular hypertrophy and systolic dysfunction: the Framingham heart study," Journal of the American Medical Association, vol. 288, no. 10, pp. 1252-1259, 2002.

[17] S. Neuhold, M. Huelsmann, G. Strunk et al., "Comparison of copeptin, b-type natriuretic peptide, and amino-terminal prob-type natriuretic peptide in patients with chronic heart failure: prediction of death at different stages of the disease," Journal of the American College of Cardiology, vol. 52, no. 4, pp. 266-272, 2008.
[18] P. Bettencourt, A. Azevedo, J. Pimenta, F. Friões, S. Ferreira, and A. Ferreira, "N-terminal-pro-brain natriuretic peptide predicts outcome after hospital discharge in heart failure patients," Circulation, vol. 110, no. 15, pp. 2168-2174, 2004.

[19] G. C. Fonarow, W. F. Peacock, T. B. Horwich et al., "Usefulness of B-type natriuretic peptide and cardiac troponin levels to predict in-hospital mortality from ADHERE," The American Journal of Cardiology, vol. 101, no. 2, pp. 231-237, 2008.

[20] A. S. Maisel, P. Krishnaswamy, R. M. Nowak et al., "Rapid measurement of B-type natriuretic peptide in the emergency diagnosis of heart failure," The New England Journal of Medicine, vol. 347, no. 3, pp. 161-167, 2002.

[21] P. Porapakkham, H. Zimmet, B. Billah, and H. Krum, "Btype natriuretic peptide-guided heart failure therapy: a metaanalysis," Archives of Internal Medicine, vol. 170, no. 6, pp. 507514, 2010.

[22] G. M. Felker, V. Hasselblad, A. F. Hernandez, and C. M. O'Connor, "Biomarker-guided therapy in chronic heart failure: a meta-analysis of randomized controlled trials," American Heart Journal, vol. 158, no. 3, pp. 422-430, 2009.

[23] M. Pfisterer, P. Buser, H. Rickli et al., "BNP-guided vs symptomguided heart failure therapy the trial of intensified vs standard medical therapy in elderly patients with congestive heart failure (TIME-CHF) randomized trial," Journal of the American Medical Association, vol. 301, no. 4, pp. 383-392, 2009.

[24] J. G. Lainchbury, R. W. Troughton, K. M. Strangman et al., "Nterminal pro- B-type natriuretic peptide-guided treatment for chronic heart failure: results from the BATTLESCARRED (NTproBNP-Assisted Treatment To Lessen Serial Cardiac Readmissions and Death) trial," Journal of the American College of Cardiology, vol. 55, no. 1, pp. 53-60, 2009.

[25] A. S. Dhaliwal, A. Deswal, A. Pritchett et al., "Reduction in BNP levels with treatment of decompensated heart failure and future clinical events," Journal of Cardiac Failure, vol. 15, no. 4, pp. 293299, 2009.

[26] P. Jourdain, G. Jondeau, F. Funck et al., "Plasma brain natriuretic peptide-guided therapy to improve outcome in heart failure. The STARS-BNP Multicenter Study," Journal of the American College of Cardiology, vol. 49, no. 16, pp. 1733-1739, 2007.

[27] I. L. Gerber, R. A. H. Stewart, M. E. Legget et al., "Increased plasma natriuretic peptide levels reflect symptom onset in aortic stenosis," Circulation, vol. 107, no. 14, pp. 1884-1890, 2003.

[28] A. Morello, D. M. Lloyd-Jones, C. U. Chae et al., "Association of atrial fibrillation and amino-terminal pro-brain natriuretic peptide concentrations in dyspneic subjects with and without acute heart failure: results from the ProBNP Investigation of Dyspnea in the Emergency Department (PRIDE) Study," American Heart Journal, vol. 153, no. 1, pp. 90-97, 2007.

[29] S. Anwaruddin, D. M. Lloyd-Jones, A. Baggish et al., "Renal function, congestive heart failure, and amino-terminal probrain natriuretic peptide measurement: results from the ProBNP investigation of dyspnea in the emergency department (PRIDE) study," Journal of the American College of Cardiology, vol. 47, no. 1, pp. 91-97, 2006.

[30] T. J. Wang, M. G. Larson, D. Levy et al., "Impact of age and sex on plasma natriuretic peptide levels in healthy adults," American Journal of Cardiology, vol. 90, no. 3, pp. 254-258, 2002.

[31] A. Bayes-Genis, D. M. Lloyd-Jones, R. R. J. Van Kimmenade et al., "Effect of body mass index on diagnostic and prognostic usefulness of amino-terminal pro-brain natriuretic peptide in patients with acute dyspnea," Archives of Internal Medicine, vol. 167, no. 4, pp. 400-407, 2007. 
[32] L. B. Daniels, P. Clopton, V. Bhalla et al., "How obesity affects the cut-points for B-type natriuretic peptide in the diagnosis of acute heart failure. Results from the Breathing Not Properly Multinational Study," American Heart Journal, vol. 151, no. 5, pp. 999-1005, 2006.

[33] T. Kerola, T. Nieminen, S. Hartikainen, R. Sulkava, O. Vuolteenaho, and R. Kettunen, "B-type natriuretic peptide as a predictor of declining cognitive function and dementia-a cohort study of an elderly general population with a 5-year follow-up," Annals of Medicine, vol. 42, no. 3, pp. 207-215, 2010.

[34] L. B. Daniels, G. A. Laughlin, D. Kritz-Silverstein et al., "Elevated natriuretic peptide levels and cognitive function in community-dwelling older adults," The American Journal of Medicine, vol. 124, no. 7, pp. 670el-670e8, 2011.

[35] J. Gunstad, A. Poppas, S. Smeal et al., "Relation of brain natriuretic peptide levels to cognitive dysfunction in adults $>55$ years of age with cardiovascular disease," The American Journal of Cardiology, vol. 98, no. 4, pp. 538-540, 2006.

[36] M. Feola, G. L. Rosso, M. Peano et al., "Correlation between cognitive impairment and prognostic parameters in patients with congestive heart failure," Archives of Medical Research, vol. 38, no. 2, pp. 234-239, 2007.

[37] L. Leto and M. Feola, "Cognitive impairment in heart failure patients," Journal of Geriatric Cardiology, vol. 11, no. 4, pp. 316328, 2014. 


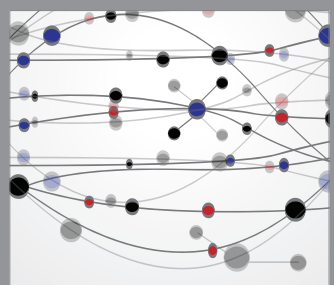

The Scientific World Journal
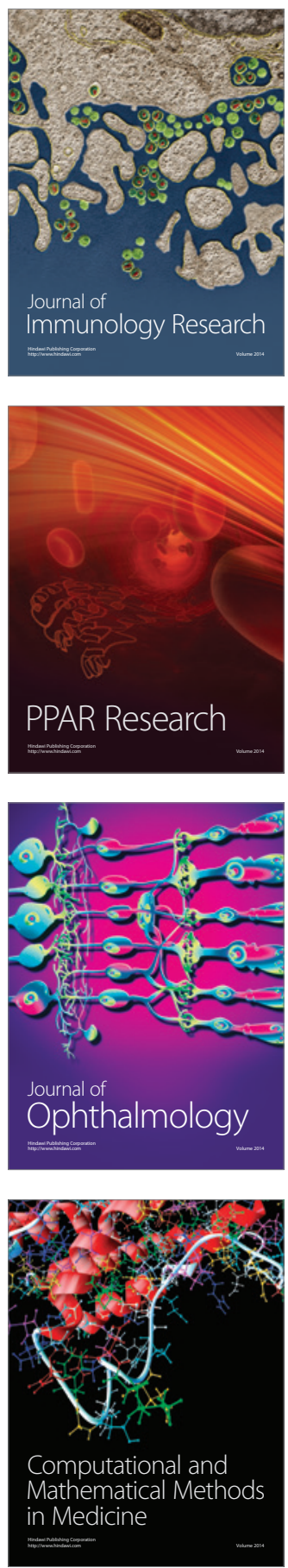

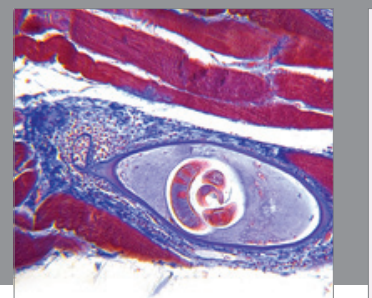

Gastroenterology

Research and Practice
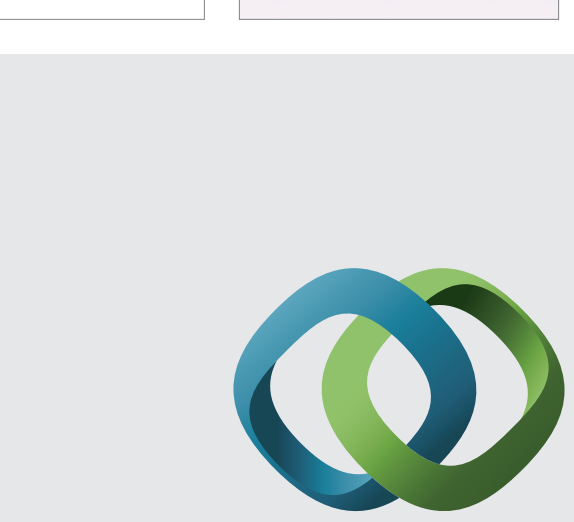

\section{Hindawi}

Submit your manuscripts at

http://www.hindawi.com
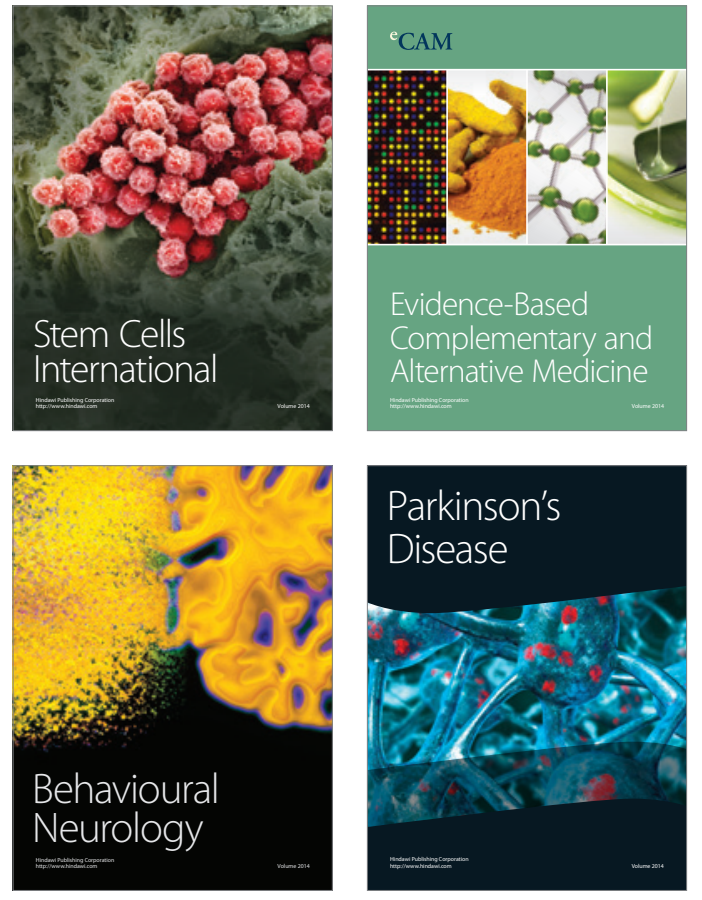
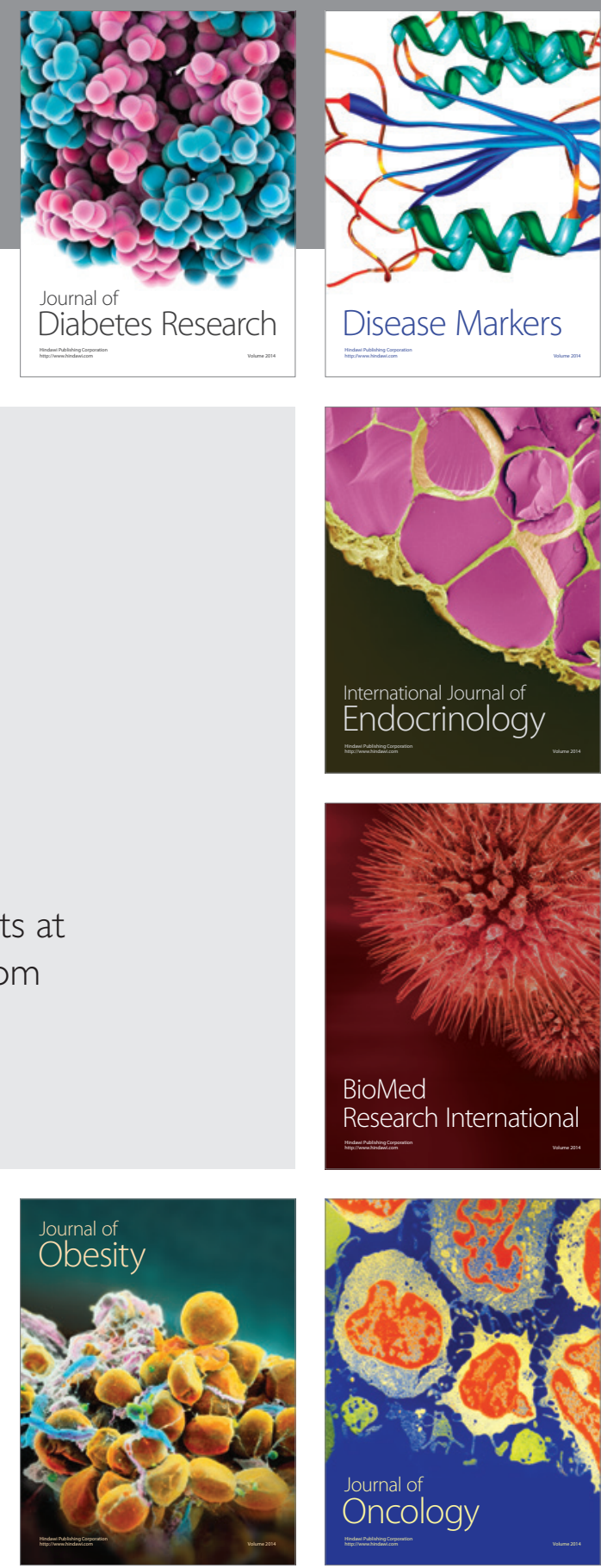

Disease Markers
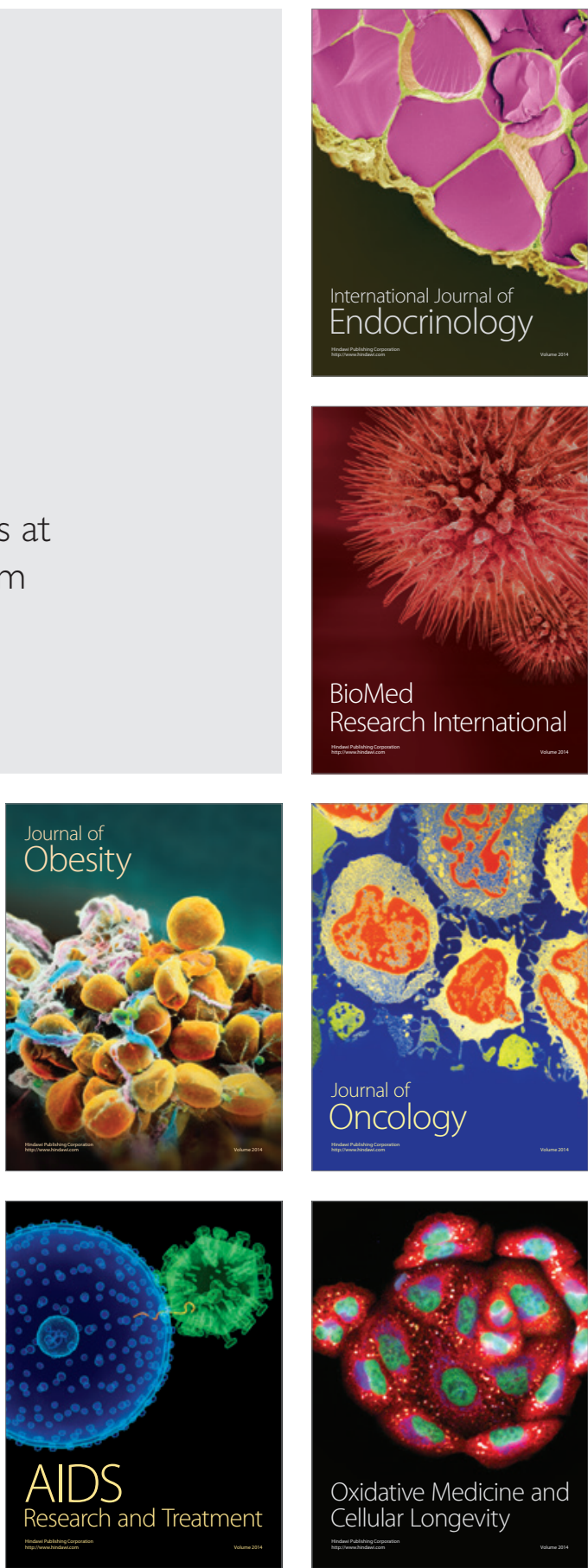